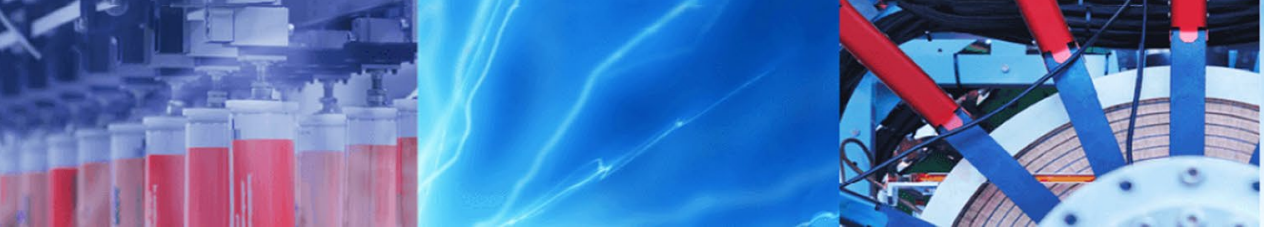

Research Article

\title{
A new graphical technique for acceleration analysis of four bar mechanisms using the instantaneous center of zero acceleration
}

\author{
Nadim Diab ${ }^{1}$ (D)
}

Received: 7 October 2020 / Accepted: 1 February 2021 / Published online: 15 February 2021

(C) The Author(s) 2021 OPEN

\begin{abstract}
In this work, a new graphical technique is furnished for acceleration analysis of four bar mechanisms through locating the instantaneous center of zero acceleration $I C_{a}$ of the coupler link. First, the paper observes the coupler's $I C_{a}$ locus and then proceeds with a series of graphical constructions that eventually lead into locating the $I_{a}$ and obtaining the linear/ angular accelerations of the coupler and follower (or slider) links. Based on the proposed graphical technique, the ease of acceleration analysis for four-bar mechanisms with varying driver's angular acceleration is demonstrated. Simultaneously, the inflection circle of the coupler curve is constructed without the need to apply Euler-Savary equations. With fewer constructions than classical graphical techniques, the robustness and simplicity of the proposed method are demonstrated by performing acceleration analysis of a slider-crank (RRRP) and a planar quadrilateral linkage (RRRR).
\end{abstract}

Keywords Planar mechanisms · Instantaneous center of zero acceleration · Inflection circle

\section{Introduction}

Understanding the kinematics of planar linkages is the first building-block in designing functional mechanisms. From the simple four bar mechanisms to the more complex higher order linkages, planar mechanisms have gained lots of attention from researchers. Investigating the performance of planar linkages covers various fields of study; from kinematic and kinetic analysis [23, 31, 50] to mechanisms synthesis $[2,9,21,22,25,26]$ and design optimization $[8,38-40,42,46]$. Understanding the behavior of these mechanisms serves in developing more complex kinematic bodies $[33,37,45]$, robots $[6,28,36]$ and grippers $[5,20,43,47,49]$. Among the most prominent kinematic properties of a planar mechanism are the locations of the instant centers of zero velocity and acceleration. For a simple four bar mechanism, the instant centers of zero velocity $\left(I C_{v}\right)$ are easily located by Aronhold-Kennedy's rule which states that the $I C_{v}$ for any three links undergoing planar motion are collinear. For higher order mechanisms whose instant centers of zero velocity are not easily detected, several techniques have been proposed in literature that can be divided into two main categories; analytical and graphical.

Yang and Hsu [51] used the loop closure equations and singularities of the coefficient matrix obtained from velocity analysis to numerically solve for the $I C_{v}$ of kinematically indeterminate systems. Di Gregorio [16] presented an analytical algorithm to locate the instant centers in 1-DOF planar mechanisms based on the linkages configuration and loop-closure equations. Kung and Wang [24] categorized the $I C_{v}$ of planar mechanisms into different levels according to their geometric dependencies. Accordingly, they recursively located the $I C_{v}$ of the planar linkages by analytically solving second and fourth order polynomial equations.

The main disadvantage of the aforementioned analytical methods is that velocities need to be calculated prior to locating the $I C_{v}$. From here rises the importance of

Nadim Diab, diabna@rhu.edu.lb| 'Department of Mechanical and Mechatronics Engineering, Rafik Hariri University, Damour, Lebanon. 
graphical methods in locating $I C_{v}$ where the visualization of the velocity fields is rendered feasible.

Dijksman [12] used a graphical method to locate instantaneous centers of zero velocity that depends on firstorder reduction through joint-joining. Later, the author developed his technique to second-order joint-joining [13] to locate the coordinated centers of curvature in a network mechanism. Zarkandi [52] presented a graphical method to locate the $I C_{v}$ of 1-DOF kinematically indeterminate spherical mechanisms. Pennock and Sankaranarayanan [29] located the instant centers of a geared seven-bar mechanism by removing one binary link from the original mechanism and thus transforming it from a 1-DOF seven bar to a 2-DOF six bar mechanism. Chang and Her [4] presented a graphical based method for locating $I C_{v}$ by introducing a translational cam in contact with one link of the kinetically indeterminate linkage. Foster and Pennock [15] proposed a new method to locate the secondary instantaneous centers of zero velocity for an eight-bar double butterfly linkage by fixing the ternary link and disconnecting the resulting 2-DOF nine-bar linkage at the coupler point. Lately, the author of this paper proposed a new graphical technique [7] based on transforming the 1-DOF mechanism into a 2-DOF counterpart by converting any ternary link adjacent to the ground into two connected binary links. Fixing each of these two binary links, one at a time, results in two different 1-DOF mechanisms where the intersection of the loci of their instantaneous centers will determine the location of the desired instantaneous center of zero velocity for the original 1-DOF mechanism.

Kinematicians have long been working on studying the characteristics and significance of the $I_{a}$ in analyzing the kinematics of planar linkages. It is worth noting at this point that the IC of zero velocity where a body appears to be in pure rotation with respect to velocity does not necessarily coincide with the IC of zero acceleration where a body appears to be in pure rotation with respect to acceleration. The $I_{a}$ has been extensively used as a point on the inflection circle to analyze the kinematics of planar motion for different systems and applications [10, 17, 44]. The importance of the inflection circle is that it contains all points on the body (or its extension) that are instantaneously undergoing a straight line path (i.e. velocity and acceleration have same direction). Several work in literature focused on constructing the inflection circle using both analytical and graphical techniques [1, 11, 19, 34] that are based on different forms of the Euler-Savary equation [3]. This commonly used equation has four different forms [48] and is used to obtain the center and radius of curvature of any point on the coupler curve known as the osculating circle $[14,18]$. The osculating circle facilitates the analysis of all properties of a planar linkage's motion up to second-order and hence is very useful in analyzing instantaneous velocities and accelerations of any point on a planar mechanism. Those specific points with infinite radius of curvature (i.e. moving instantaneously on a straight line path) define the inflection circle. Pennock and Kinzel [30] proposed a new method, independent of the Euler-Savary equation, to find the center and radius of curvature of a coupler point trajectory by attaching a virtual link from the coupler point to the center of its corresponding osculating circle.

While all the aforementioned techniques are useful in constructing the inflection circle for the coupler linkage and the osculating circle for the coupler point path of a four bar mechanism, locating the specific point on the former circle corresponding to the $I C_{a}$ needs additional graphical constructions as discussed by Schiller [35]. These graphical constructions are dependent on the angular velocity and acceleration of different links of the planar linkage in addition to its position. This is inevitable since the $I C_{a}$ is dependent on both, the linkages' angular velocities/accelerations and positions, whereas the inflection and osculating circles are solely dependent on the latter. Having located the $I C_{a}$, getting the acceleration of the coupler/follower joint for RRRR mechanisms or the slider acceleration for RRRP mechanisms needs further implementation of some readily available basic analytical techniques. Sun and Liu [41] presented an analytical method for locating the $I C_{a}$ for a rigid body in plane motion. Their method is based on applying some mathematical manipulations (vector algebra) of the relative acceleration between any two points on the planar body to prove the existence of the $I C_{a}$ and to calculate its position. However, the coordinates of the $I C_{a}$ were determined as a function of the angular acceleration of the body itself which is usually unknown prior to acceleration analysis of the coupler link in four bar mechanisms. Therefore, the method of [41] can locate the $I C_{a}$ merely after performing an acceleration analysis over the four bar mechanism and does not benefit from the unique features of the $I C_{a}$ to conduct acceleration analysis on planar linkages.

In this work, a genuine graphical method is proposed to perform acceleration analysis of a four bar mechanism based on the unique location of the $I_{a}$. The proposed method requires appreciably fewer graphical constructions than techniques previously reported in literature and doesn't require prior construction of the inflection circle for the coupler linkage. In case the inflection circle is to be constructed after locating the $I C_{a}$, this can be done in a straightforward manner without the need to use the Euler-Savary equation. Two points of that circle are readily available (i.e. the $I C_{v}$ and $I C_{a}$ ) while the third point on the inflection circle diametrically opposite to the $I C_{v}$, known as inflection pole, can be located in one simple graphical construction as will be demonstrated 
later in this paper. Most importantly, the method formulated in this work not only locates the $I C_{a}$, but also obtains the acceleration of the coupler/follower joint or slider simultaneously.

The following section reviews the properties of the unique location of the instantaneous center of zero acceleration for planar mechanisms. It is followed in sect. 3 by the proposed graphical methodology that details the formulations of the genuine graphical technique adopted in this work. Following this formulation are two case studies to demonstrate the ease of use of the proposed graphical technique in acceleration analysis of four bars; the first addresses a slider crank (RRRP) mechanism while the second addresses a quadrilateral linkage (RRRR). Section 6 illustrates the exploitation of the graphical technique proposed in this study for acceleration analysis of a four-bar mechanism with varying driver's angular acceleration. All results obtained are discussed in sect. 7 and conclusions extracted from this work are furnished in the last section.

\section{Instantaneous center of zero acceleration}

In this section, the location of the instantaneous center of zero acceleration $\left(I C_{a}\right)$ is revisited where the angle between the acceleration vector of any point on a planar linkage and the line joining that point to the $I_{a}$ is derived analytically. As shown in Fig. 1, let $A B$ be a planar linkage undergoing complex motion (with angular velocity $\omega_{A B}$ and angular acceleration $\alpha_{A B}$ ) and $Z$ represents its $I C_{a}$. In what follows, the angle $(\psi)$ between the acceleration vectors $\overrightarrow{a_{A}}$ and $\overrightarrow{a_{B}}$ with $\overrightarrow{A Z}$ and $\overrightarrow{B Z}$ respectively will be derived.

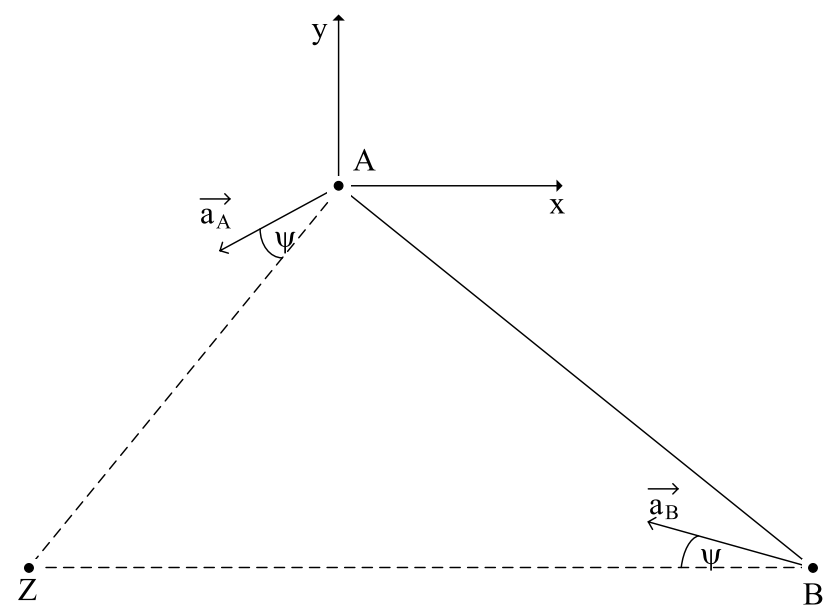

Fig. 1 Location of the instantaneous center of zero acceleration for a planar linkage
For the coordinate system $A-x y z$ with unit vectors $\hat{x}, \hat{y}$ and $\hat{z}$ :

$\frac{y_{Z}}{x_{Z}}=\tan (\angle \overrightarrow{A Z})=\tan \left(\angle \overrightarrow{a_{A}}+\psi\right)=\frac{\frac{a_{A y}}{a_{A x}}+\tan (\psi)}{1-\tan (\psi) \frac{a_{A y}}{a_{A x}}}$

The relative acceleration equation between points $A$ and $Z$ is given by:

$\overrightarrow{a_{A}}=\overrightarrow{a_{Z}}+\overrightarrow{a_{A / Z}}$

But $\overrightarrow{a_{z}}=0$,

$$
\begin{aligned}
& \Longrightarrow a_{A x} \hat{x}+a_{A y} \hat{y}=\alpha_{A B} \hat{z} \times\left(\left(x_{A}-x_{Z}\right) \hat{x}+\left(y_{A}-y_{Z}\right) \hat{y}\right) \\
& -\omega_{A B}^{2}\left(\left(x_{A}-x_{Z}\right) \hat{x}+\left(y_{A}-y_{Z}\right) \hat{y}\right)
\end{aligned}
$$

Since $A$ is the origin of our coordinate system, $x_{A}=y_{A}=0$, therefore solving the above equation for $x_{Z}$ and $y_{Z}$ gives:

$x_{Z}=\frac{\omega_{A B}^{2} a_{A x}-\alpha_{A B} a_{A y}}{\alpha_{A B}^{2}+\omega_{A B}^{4}}$

$y_{Z}=\frac{\omega_{A B}^{2} a_{A y}+\alpha_{A B} a_{A x}}{\alpha_{A B}^{2}+\omega_{A B}^{4}}$

Substituting Eqs. 4 and 5 in Eq. 1 gives:

$\tan (\psi)=\frac{\alpha_{A B}}{\omega_{A B}^{2}}$

\section{Proposed graphical methodology}

For a classical acceleration analysis problem of a 4-bar mechanism, all link dimensions in addition to the angular velocity and acceleration of the driver link are known and it is required to get the angular acceleration of the coupler and follower links in addition to the acceleration of the coupler-follower joint. For a slider-crank mechanism, the angular acceleration of the coupler in addition to the linear acceleration of the slider are solved for. The classical approach in solving such problems is to perform position analysis (i.e. get coupler and follower orientations) by either graphical or analytical methods. This is followed by velocity analysis (i.e. get coupler and follower angular velocities) which is also performed by either graphical or analytical methods. The graphical method is commonly divided into two techniques; the first depends on the velocity polygon while the second uses the $I C_{v}$. Finally to perform acceleration analysis, graphical and analytical techniques are also used where the most common technique for the graphical method is the acceleration 


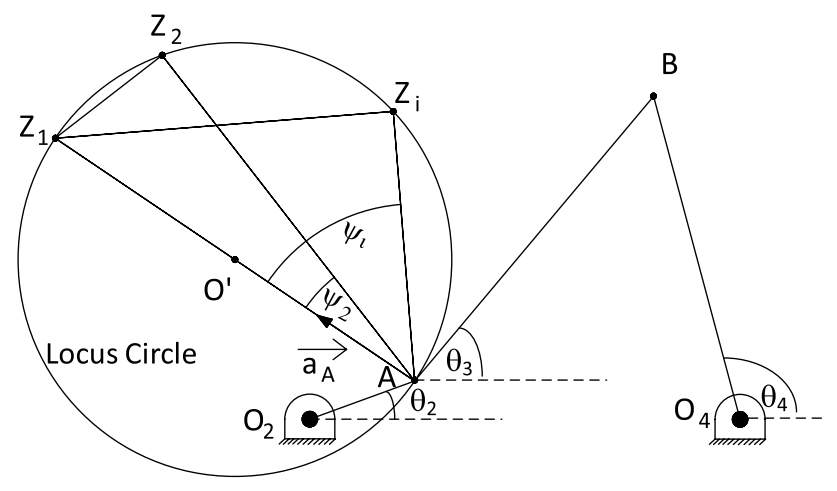

Fig. 2 Circle traced by the coupler's $C_{a}$ for varying coupler's angular acceleration

polygon. As discussed earlier in the introduction section, the graphical methods for performing acceleration analysis that take advantage of the unique features of the $I C_{a}$ are relatively lengthy and incomprehensive in finding the acceleration of the coupler-follower joint or slider as they require additional analytical computations and/or graphical constructions. In what follows, a new graphical methodology for simultaneously performing acceleration analysis and locating the $I C_{a}$ of RRRR and RRRP mechanisms is furnished. Figure 2 displays a 4R mechanism where the ground, driver, coupler and follower links are numbered as 1,2, 3 and 4 respectively. Accordingly, the orientations, angular velocities and angular accelerations of the ground, driver, coupler and follower are given by $\left(\theta_{1}, \theta_{2}, \theta_{3}, \theta_{4}\right),\left(\omega_{1}\right.$, $\left.\omega_{2}, \omega_{3}, \omega_{4}\right)$ and $\left(\alpha_{1}, \alpha_{2}, \alpha_{3}, \alpha_{4}\right)$ respectively. This terminology will be adopted all throughout this work. If $Z$ is the point locating the $I C_{a}$ of the coupler link $A B$, then the coupler appears to be in pure rotation with respect to point $Z$ (strictly from an acceleration point of view) and consequently the normal component of the relative acceleration of point $A$ with respect to $Z$ is given as:

$a_{A / Z}^{n}=\left(a_{A}\right) \cos (\psi)=(\overline{Z A}) \omega_{3}^{2}$

$\Longrightarrow \overline{Z A}=\left(\frac{a_{A}}{\omega_{3}^{2}}\right) \cos (\psi)$

In the above equation, the location of point $A$ is known and the values of $a_{A}$ and $\omega_{3}$ are available (the latter is obtained through the velocity analysis phase). The two unknowns are the location of the coupler's $I C_{a}$ (i.e. point $Z$ ) and $\psi$. It is obvious that as the angular velocity $\left(\omega_{2}\right)$ and/ or angular acceleration $\left(\alpha_{2}\right)$ of the driver link change, the angular acceleration of the coupler link $\left(\alpha_{3}\right)$ changes accordingly and so does $\psi$ since $\tan (\psi)=\frac{\alpha_{3}}{\omega_{3}^{2}}$. Therefore, for the $4 \mathrm{R}$ mechanism in Fig. 2, varying the angular

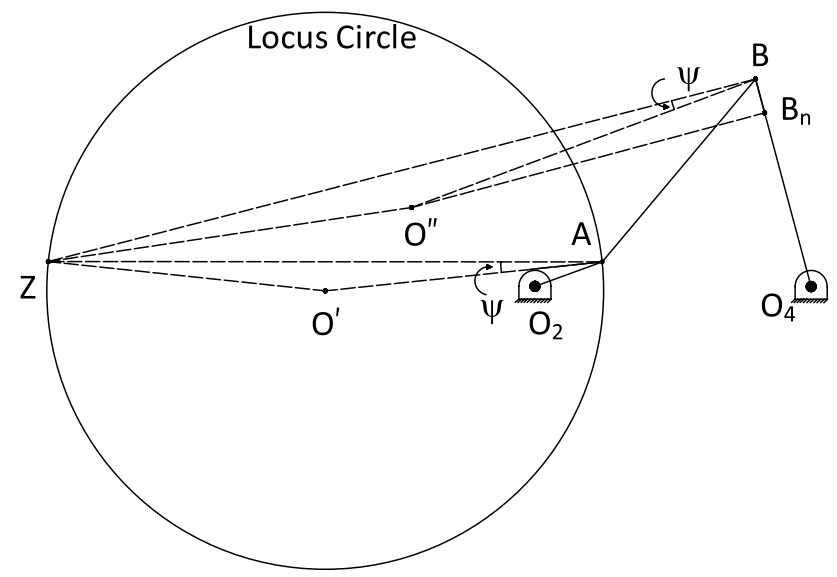

Fig. 3 Unique location of the coupler's $I C_{a}$ on the traced circle of Fig. 2

acceleration of the coupler link will change the location of the coupler's instantaneous center of zero acceleration $\left(I C_{a}\right)$ in a pattern to be yet determined from Eq. 8.

Assume $\psi_{1}=0$, then the coupler's $I C_{a}$ given by $Z_{1}$ is a point along $\overrightarrow{a_{A}}$ and at a distance $\frac{a_{A}}{\omega_{3}^{2}}$ from $A$. If we vary the angular acceleration of the coupler link, the new position of the coupler's $I_{a}$ (i.e. point $Z_{2}$ ) will be at a distance $\overline{Z_{2} A}=\left(\overline{Z_{1} A}\right) \cos \left(\psi_{2}\right)$ from $A$ and at angle $\psi_{2}$ from $\overline{A Z_{1}}$. In general, for any value of the yet unknown angular acceleration of the coupler link $\left(\alpha_{3}\right)$, the location of its instantaneous center of zero acceleration (i.e. point $Z_{i}$ ) is located by:

$\overline{Z_{i} A}=\left(\overline{Z_{1} A}\right) \cos \left(\psi_{i}\right)=\left(\frac{a_{A}}{\omega_{3}^{2}}\right) \cos \left(\psi_{i}\right)$

where $Z_{i}$ is the locus of the coupler's $I C_{a}$ representing all possible locations of point $Z$ for different coupler's angular accelerations.

From Eq. 9, it can be concluded that $\angle Z_{1} Z_{i} A=\frac{\pi}{2}$ (Fig. 2) and therefore:

For varying coupler's angular acceleration, the instantaneous center of zero acceleration of the coupler link of a four bar mechanism traces a circle of center $O^{\prime}$ located along the direction of $\overrightarrow{a_{A}}$ and radius $\overrightarrow{O^{\prime} A}=\frac{a_{A}}{2 \omega_{3}^{2}}$.

It is important to mention at this point that the aforementioned circle does not define the inflection circle of the coupler curve and will be referred to as locus circle in this study. In fact, as will be demonstrated later, the $I_{a}$ is the intersection point between the circle in Fig. 2 and the coupler's instantaneous inflection circle. Next step is to locate the exact point on circle of Fig. 2 that corresponds to the coupler's instantaneous center of zero acceleration for a specific angular acceleration $\left(\alpha_{3}\right)$. This specific value of the coupler's angular acceleration is the kinematic 
analysis solution corresponding to the pre-specified angular acceleration $\left(\alpha_{2}\right)$ of the driver link. As shown in Fig. 3, let's assume $Z$ to be the $I C_{a}$ of the coupler. By referring back to Fig. 1 and Eq. 9, similar analogy can be conducted on point $\mathrm{B}$ of the coupler where $\left(a_{B}\right) \cos (\psi)=(\overline{Z B}) \omega_{3}^{2}$ and therefore the instantaneous center of zero acceleration of the coupler link traces a circle of center $O^{\prime \prime}$ located along the direction of $\overrightarrow{a_{B}}$ and radius $\overrightarrow{O^{\prime \prime} B}=\frac{a_{B}}{2 \omega_{3}^{2}}$. Accordingly, $\overrightarrow{B O^{\prime \prime}}$ defines the acceleration vector of point $B$ (i.e. $\overrightarrow{a_{B}}$ ) where its projection on the follower $\overline{B B_{n}}=\frac{a_{B}^{n}}{2 \omega_{3}^{2}}=\frac{\left.\omega_{4}^{2} \overline{\bar{O}_{4} B}\right)}{2 \omega_{3}^{2}}$. This is very important since $a_{B^{\prime}}^{n}$ the normal component of $\overrightarrow{a_{B}}$, is readily available following the velocity analysis phase. Figure 3 shows that $\triangle Z O^{\prime \prime} B$ is similar to $\triangle Z O^{\prime} A$ since $\angle O^{\prime} A Z=\angle O^{\prime} Z A=\angle O^{\prime \prime} B Z=\angle O^{\prime \prime} Z B=\psi$. Therefore, $\triangle Z O^{\prime} O^{\prime \prime}$ is similar to $\triangle Z A B$ since $\frac{\overline{Z O^{\prime}}}{\overline{Z O^{\prime \prime}}}=\frac{\overline{Z A}}{\overline{Z B}}$ and $\angle O^{\prime} Z O^{\prime \prime}=\angle A Z B$. From here, it can be concluded that the angle between $\overline{O^{\prime} O^{\prime \prime}}$ and the coupler link $\overline{A B}$ is nothing but $\psi$, the angle between $\overline{O^{\prime} Z}$ and $\overline{Z A}$ (i.e. $\angle O^{\prime} Z A$ ) and the angle between $\overline{O^{\prime \prime} Z}$ and $\overline{B Z}$ (i.e. $\angle O^{\prime \prime} Z B$ ). From the same pair of similar triangles, it can concluded that $\frac{\overline{O^{\prime} O^{\prime \prime}}}{\overline{A B}}=\frac{\overline{Z O^{\prime \prime}}}{\overline{Z B}}=\frac{\overline{O^{\prime \prime} B}}{\overline{Z B}} \Longrightarrow \overline{O^{\prime} O^{\prime \prime}}=\frac{\left(\overline{O^{\prime \prime} B}\right)(\overline{A B})}{\overline{Z B}} \operatorname{but}\left(\overline{O^{\prime \prime} B}\right) \cos \psi=\frac{\overline{Z B}}{2}$ therefore,

$\left(\overline{O^{\prime} O^{\prime \prime}}\right) \cos \psi=\frac{\overline{A B}}{2}$

The last equation is the key for the proposed graphical method as it states:

The projection of $\overline{O^{\prime} O^{\prime \prime}}$ on the coupler $A B$ with which it makes an angle $\psi$ is $\frac{\overline{A B}}{2}$.

All of the above will be summarized in the following steps needed to perform acceleration analysis of four-bar mechanisms and will be illustrated in Fig. 4:

(1) Plot a circle of center $O^{\prime}$ along $\overrightarrow{a_{A}}$ where $\overline{O^{\prime} A}=\frac{a_{A}}{2 \omega_{3}^{2}}$

(2) Copy coupler $\overline{A B}$ from point $A$ and paste it at point $O^{\prime}$ while keeping its orientation fixed (i.e. $\overline{O^{\prime} B^{\prime}}$ )

(3) Draw the perpendicular bisector of the relocated coupler link $\left(\overline{O^{\prime} B^{\prime}}\right)$ and intersect it with the normal dropped from point $B_{n}$ located at distance $\frac{\omega_{4}^{2}\left(\overline{O_{4} B}\right)}{2 \omega_{3}^{2}}$ from

B. The intersection of the two normals is point $O^{\prime \prime}$. For a slider-crank mechanism, the perpendicular bisector of the relocated coupler link is intersected with the line along the sliding direction (i.e. along $\overrightarrow{a_{B}}$ ) to give point $O^{\prime \prime}$

(4) The angle obtained between $\overline{O^{\prime} B^{\prime}}$ and $\overline{O^{\prime} O^{\prime \prime}}$ is $\psi$ and therefore the intersection of the circle of center $O^{\prime \prime}$ and radius $\overline{O^{\prime \prime} B}$ with the circle of center $O^{\prime}$ and radius $\overline{O^{\prime} A}$ locates the $I C_{a}$ (i.e. point $Z$ ) of the coupler link.

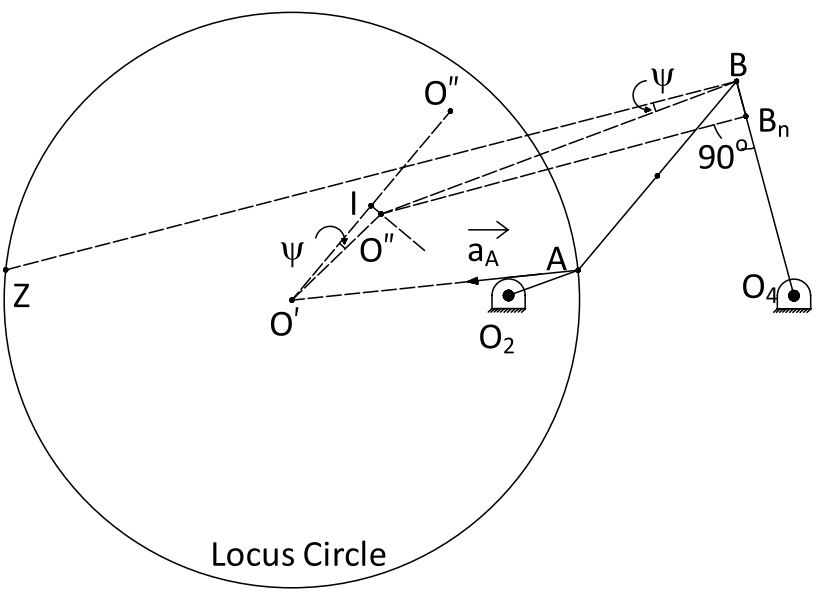

Fig. 4 Proposed graphical method to locate the $I C_{a}$ of the coupler's link of a planar mechanism

The above listed four steps will give all the information needed for the acceleration analysis of the $4 R$ mechanism in Fig. 4. For instance, the acceleration of joint $B \overrightarrow{a_{B}}=\frac{\overrightarrow{B O^{\prime \prime}}}{2 \omega_{3}^{2}}$, its tangential acceleration $\overrightarrow{a_{B}^{t}}=\frac{\overrightarrow{B_{n} 0^{\prime \prime}}}{2 \omega_{3}^{2}}$, angular acceleration of coupler link $\alpha_{3}=\tan (\psi) \omega_{3^{\prime}}^{2}$ and angular acceleration of follower link $\alpha_{4}=\frac{a_{B}^{t}}{\bar{O}_{4} B}$. In addition, the coupler's instantaneous center of zero acceleration (i.e. point $Z$ ) can be located by simply rotating $\overrightarrow{B O^{\prime \prime}}$ through angle $\psi$ and intersecting it with the circle of step 1. Moreover, the inflection circle can be easily constructed since two of its points are already available (the instantaneous centers of zero velocity (call it point $P$ ) and acceleration (i.e. $Z$ ) for the coupler link). For the slider-crank mechanism, the coupler/slider joint constitutes the third point of the inflection circle as it undergoes a straight line motion. For the $4 \mathrm{R}$ mechanism, the third point can be located by one of two methods, graphical or analytical. The first graphical method uses Juokowski theorem [32] which states that the angle between the relative acceleration vector of any two points and the line joining these two points is equal to the angle between the acceleration vector of any point and the line joining that point to the $I_{a}$. Accordingly, the acceleration vector of the coupler's $/ C_{v}$ (i.e. point $P$ ) makes an angle $\psi$ with line $\overline{P Z}$ and the intersection of this line with the velocity vector dropped from point $Z$ (i.e. line normal to $\overline{P Z}$ ) locates the third point on the inflection circle $(Q)$ diametrically opposite to $P$. It is worthnoting that point $Q$ belongs to the centrode normal and is referred to in literature as the inflection pole. The second method is analytical and based on the quadratic form of the Euler-Savary equation given by: 
$\rho=\frac{\overline{R P}}{\overline{R l_{R}}}$

where $\rho$ is the radius of curvature of any point $R$ on the inflection circle and $I_{R}$ is the intersection of line $R P$ with the inflection circle. This can be used at any of the two joints of the $4 R$ mechanism's coupler link since these two points undergo a circular trajectory with known centers and radii of trajectory. Therefore, Eq. 11 can be applied to the joint connecting the driver and coupler links to get the third point on the inflection circle i.e. $I_{R}$. This work concentrates on graphical methods to locate instantaneous centers and construct inflection circles. Accordingly, the former graphical method presented earlier will be implemented.

\section{Case study I: slider crank mechanism}

Consider the slider crank mechanism shown in Fig. 5 where the driver length is $1 \mathrm{~m}$ at an angle of $30^{\circ}$ with the horizontal ground and the coupler length is $1.5 \mathrm{~m}$. The instantaneous angular velocity and angular acceleration of the driver link are $5 \mathrm{rad} / \mathrm{sec}$ and $20 \mathrm{rad} / \mathrm{sec}^{2}$ respectively. All positive values of angular velocities and accelerations in this work are considered in the counter clockwise direction. In what follows, acceleration analysis will be performed analytically starting with the classical relative velocity equation between the two coupler joints (i.e. points $B$ and $C$ ):

$$
\begin{aligned}
\overrightarrow{V_{C}}= & \overrightarrow{V_{B}}+\overrightarrow{V_{C / B}} \\
\Longrightarrow & V_{C} \vec{i}=\omega_{2}(\overline{A B}) \sin \left(\theta_{2}\right) \vec{i}-\omega_{2}(\overline{A B}) \cos \left(\theta_{2}\right) \vec{j} \\
& +\omega_{3}(\overline{B C}) \sin \left(\theta_{3}\right) \vec{i}+\omega_{3}(\overline{B C}) \cos \left(\theta_{3}\right) \vec{j}
\end{aligned}
$$

The vector equation above constitutes 2 algebraic equations that can be solved to get the desired coupler's angular velocity (i.e. $\left.\omega_{3}\right)$ :

$\omega_{3}=\frac{\omega_{2}(\overline{A B}) \cos (\theta 2)}{(\overline{B C}) \cos (\theta 3)}=3.062 \mathrm{rad} / \mathrm{sec}$

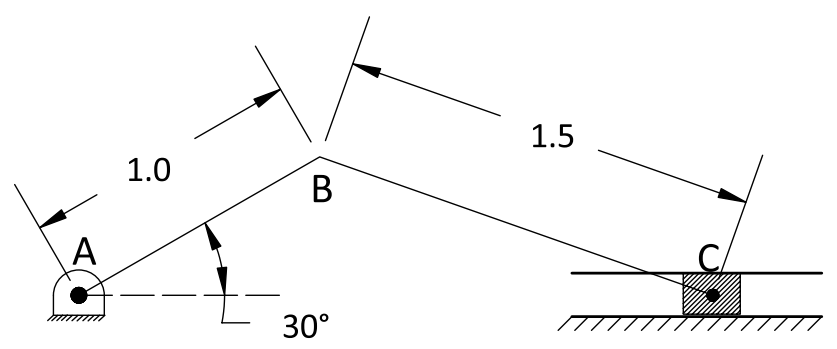

Fig. 5 Slider crank mechanism
Similarly, relative acceleration is performed between the two coupler joints:

$$
\begin{aligned}
\overrightarrow{a_{C}}= & \overrightarrow{a_{B}}+\overrightarrow{a_{C / B}} \\
\Longrightarrow & a_{C} \vec{i}=-\omega_{2}^{2}(\overline{A B}) \cos \left(\theta_{2}\right) \vec{i}-\omega_{2}^{2}(\overline{A B}) \sin \left(\theta_{2}\right) \vec{j} \\
& +\alpha_{2}(\overline{A B}) \sin \left(\theta_{2}\right) \vec{i}-\alpha_{2}(\overline{A B}) \cos \left(\theta_{2}\right) \vec{j}- \\
& \omega_{3}^{2}(\overline{B C}) \cos \left(\theta_{3}\right) \vec{i}+\omega_{3}^{2}(\overline{B C}) \sin \left(\theta_{3}\right) \vec{j} \\
& +\alpha_{3}(\overline{B C}) \sin \left(\theta_{3}\right) \vec{i}+\alpha_{3}(\overline{B C}) \cos \left(\theta_{3}\right) \vec{j}
\end{aligned}
$$

The vector equation above constitutes 2 algebraic equations that can be solved for the unknown angular acceleration of the coupler and the acceleration of the slider: $\alpha_{3}=17.772 \mathrm{rad} / \mathrm{sec}^{2}$ and $a_{\mathrm{C}}=-16.023 \mathrm{~m} / \mathrm{sec}^{2}$.

Next, acceleration analysis of the slider crank will be performed through the proposed graphical method by following steps 1 through 4 listed in the previous section as seen in Fig. 6 :

(1) $\overline{O^{\prime} B}=\frac{a_{B}}{2 \omega_{3}^{2}}=\frac{\sqrt{\left(\omega_{2}^{2}(\overline{A B})\right)^{2}+\left(\alpha_{2}(\overline{A B})\right)^{2}}}{2 \omega_{3}^{2}}=1.708 \mathrm{~m} . \quad \overrightarrow{a_{B}}$ makes an angle of $38.66^{\circ}$ (i.e. $\tan ^{-1}\left(\frac{\alpha_{2}}{\omega_{2}^{2}}\right)$ ) with $\overrightarrow{A B} \Longrightarrow O^{\prime}$ lies at a distance of $1.708 \mathrm{~m}$ from $B$ along the direction of $\overrightarrow{a_{B}}$.

(2) Copy coupler $\overline{B C}$ from point $B$ and paste at $O^{\prime}$. Call it $\overline{O^{\prime} C^{\prime}}$ and sketch its perpendicular bisector.

(3) Intersect the perpendicular bisector from step 2 with the slider direction (i.e. $\overline{A C}$ ) to locate $O^{\prime \prime}$.

4) Angle between $\overline{O^{\prime} C^{\prime}}$ and $\overline{O^{\prime} O^{\prime \prime}}$ gives $\psi=62.19^{\circ}$.

Now all acceleration analysis data are available. For example, we can get the angular acceleration of the

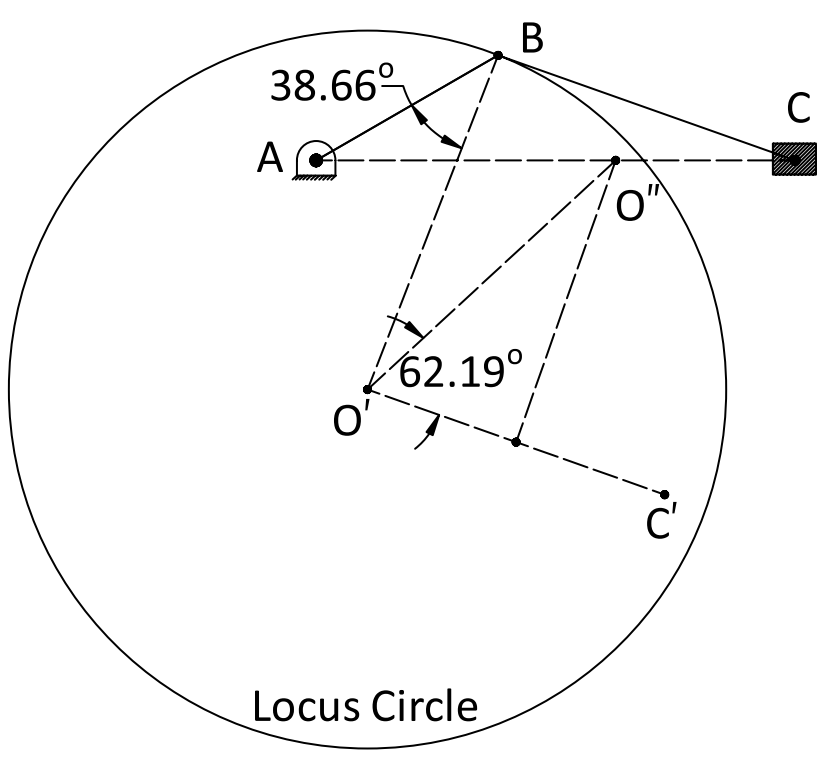

Fig. 6 Graphical constructions to determine $\psi$ for the slider crank mechanism 
coupler link $(B C)$ as: $\alpha_{3}=\tan (\psi) \omega_{3}^{2}=17.774 \mathrm{rad} / \mathrm{sec}^{2}$. The magnitude of the acceleration of the slider can be simply measured from Fig. 6 as $a_{C}=\left(2 \omega_{3}^{2}\right) \overline{C O^{\prime \prime}}=2\left(3.062^{2}\right)(0.855)=16.022 \mathrm{~m} / \mathrm{sec}^{2}$ directed to the left as indicated by $\overline{\mathrm{CO}^{\prime \prime}}$.

The obtained results from the analytical and proposed graphical methods perfectly agree. The instantaneous center of zero acceleration of the coupler link (i.e point $Z$ ) is readily available by rotating the acceleration vector of the driver/coupler joint (i.e. point $B$ ) given along $\overrightarrow{B O^{\prime}}$ or the acceleration vector of the slider given along $\overline{C^{\prime \prime}}$ by an angle $\psi=62.19^{\circ}$. The same result can be achieved by intersecting the circle of center $O^{\prime \prime}$ and radius $\overline{O^{\prime \prime} C}$ with the circle of center $O^{\prime}$ and radius $\overline{O^{\prime} B}$. All aforementioned constructions are shown in Fig. 7.

Moreover, the inflection circle can now be easily constructed (see Fig. 8) as it passes through the instantaneous center of zero velocity of the coupler link (i.e. point $P$ ), the instantaneous center of zero acceleration of the coupler link (i.e. point $Z$ ) and the coupler/follower joint (i.e. point C).

\section{Case study II: four bar mechanism}

In what follows, the four-bar mechanism in Fig. 9 is considered for acceleration analysis. All link dimensions in addition to the driver's orientation $\left(\theta_{2}\right)$, angular velocity $\left(\omega_{2}\right)$, and angular acceleration $\left(\alpha_{2}\right)$ are given: $\overline{O_{2} A}=30 \mathrm{~mm}, \overline{A B}=100 \mathrm{~mm}, \overline{O_{4} B}=90 \mathrm{~mm}, \theta_{2}=20^{\circ}$,

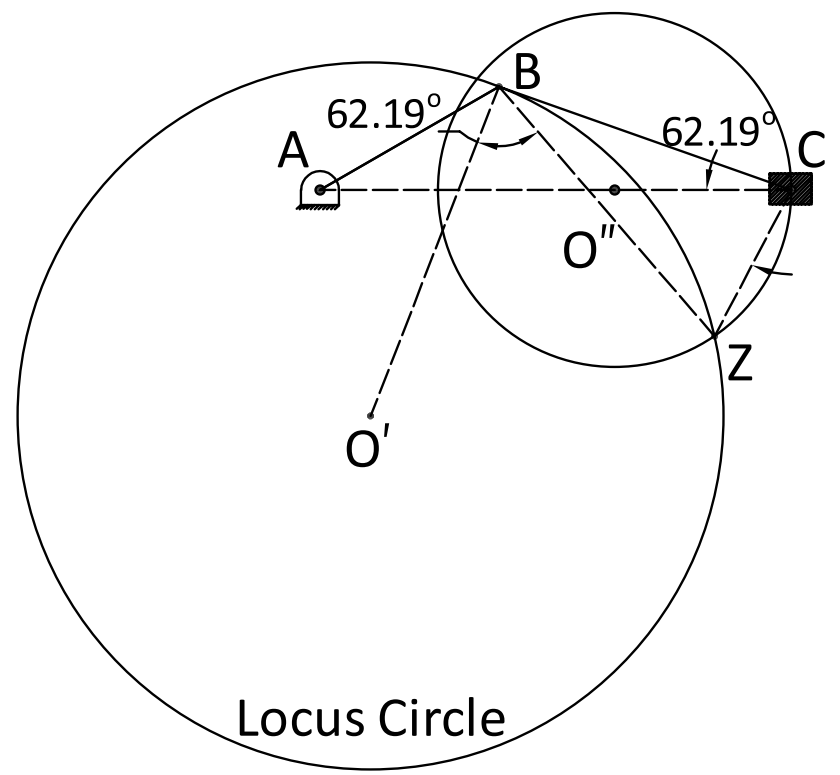

Fig. 7 Graphical constructions to determine the location of the $I C_{a}$ for the slider crank mechanism

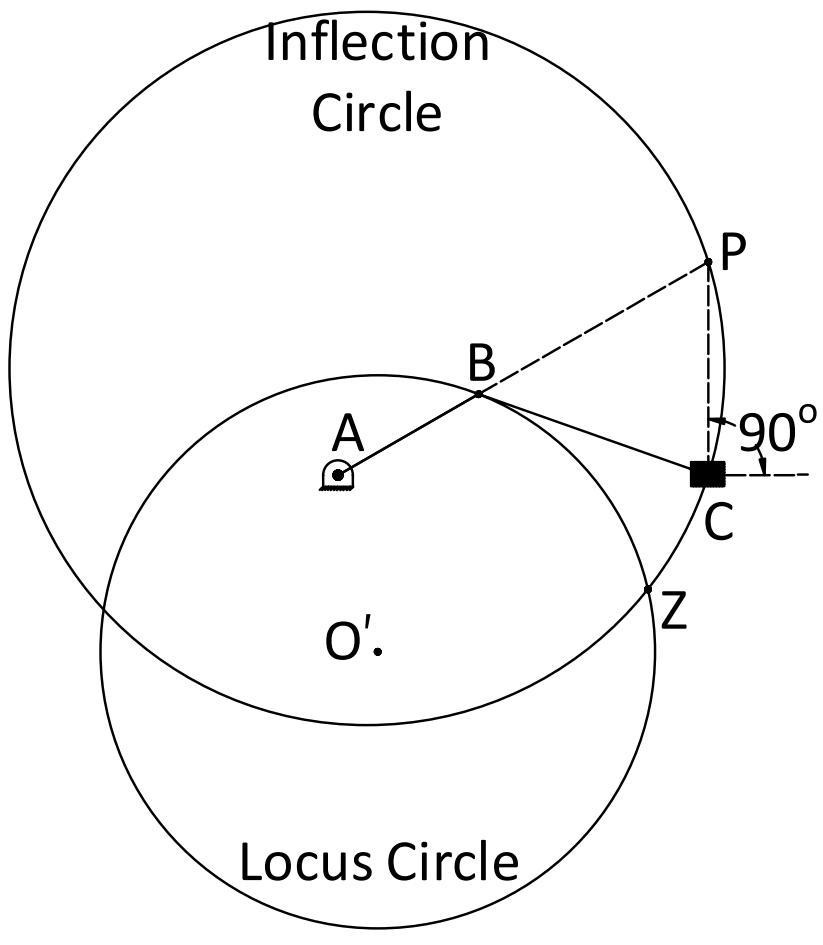

Fig. 8 Inflection circle of the slider crank mechanism

$\omega_{2}=10 \mathrm{rad} / \mathrm{sec}$, and $\alpha_{2}=25 \mathrm{rad} / \mathrm{sec}^{2}$. The orientation of the coupler $\left(\theta_{3}\right)$ and follower $\left(\theta_{4}\right)$ links can be easily measured to give $50^{\circ}$ and $75^{\circ}$ respectively. The classical analytical method will be first used to find the angular accelerations of the coupler $\left(\alpha_{3}\right)$ and follower $\left(\alpha_{4}\right)$ links in addition to the acceleration of the coupler/follower joint (i.e. point $B$ ).

Starting with the relative velocity equation:

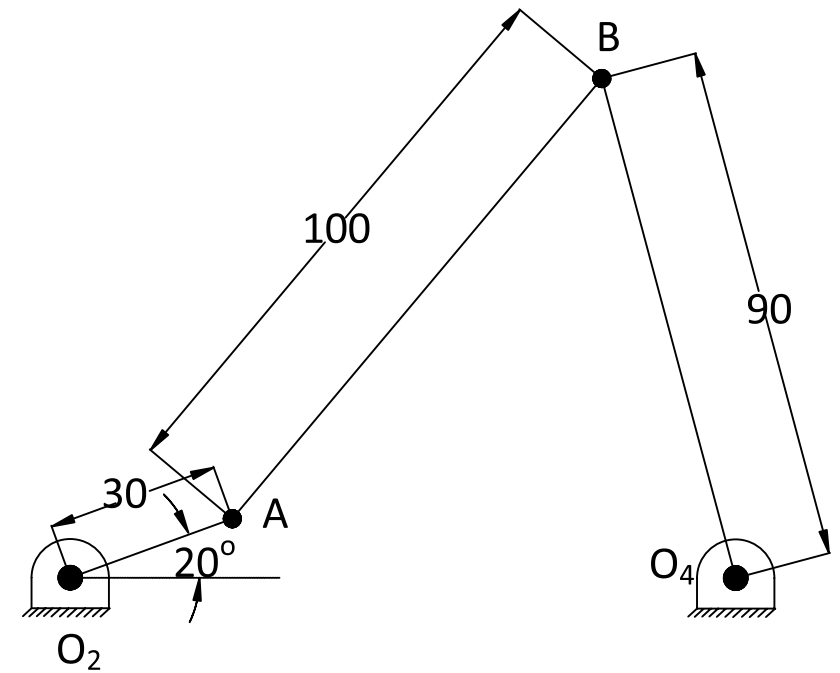

Fig. 9 Four bar mechanism 


$$
\begin{aligned}
\overrightarrow{V_{B}}= & \overrightarrow{V_{A}}+\overrightarrow{V_{B / A}} \\
\Longrightarrow & \omega_{4}\left(\overrightarrow{O_{4} B}\right) \sin \left(\theta_{4}\right) \vec{i}+\omega_{4}\left(\overrightarrow{O_{4} B}\right) \cos \left(\theta_{4}\right) \vec{j}= \\
& -\omega_{2}\left(\overline{O_{2} A}\right) \sin \left(\theta_{2}\right) \vec{i}+\omega_{2}\left(\overline{O_{2} A}\right) \cos \left(\theta_{2}\right) \vec{j} \\
& -\omega_{3}(\overline{A B}) \sin \left(\theta_{3}\right) \vec{i}+\omega_{3}(\overline{A B}) \cos \left(\theta_{3}\right) \vec{j}
\end{aligned}
$$

The vector equation above constitutes 2 algebraic equations that can be solved to get the desired coupler's and follower's angular velocities as $\omega_{3}=-3.648 \mathrm{rad} / \mathrm{sec}$ and $\omega_{4}=2.035 \mathrm{rad} / \mathrm{sec}$.

Similarly, relative acceleration is performed between the two coupler joints:

$$
\begin{aligned}
\overrightarrow{a_{B}} & =\overrightarrow{a_{A}}+\overrightarrow{a_{B / A}} \\
& \Longrightarrow \overrightarrow{a_{B}^{n}}+\overrightarrow{a_{B}^{t}}-\overrightarrow{a_{A}^{n}}-\overrightarrow{a_{A}^{t}}-\overrightarrow{a_{B / A}^{n}}-\overrightarrow{a_{B / A}^{t}}=0 \\
& \Longrightarrow \omega_{4}^{2}\left(\overrightarrow{O_{4} B}\right) \cos \left(\theta_{4}\right) \vec{i}-\omega_{4}^{2}\left(\overrightarrow{O_{4} B}\right) \sin \left(\theta_{4}\right) \vec{j} \\
& +\alpha_{4}\left(\overrightarrow{O_{4} B}\right) \sin \left(\theta_{4}\right) \vec{i}+\alpha_{4}\left(\overrightarrow{O_{4} B}\right) \cos \left(\theta_{4}\right) \vec{j} \\
& +\omega_{2}^{2}\left(\overline{O_{2} A}\right) \cos \left(\theta_{2}\right) \vec{i}+\omega_{2}^{2}\left(\overline{O_{2} A}\right) \sin \left(\theta_{2}\right) \vec{j} \\
& +\alpha_{2}\left(\overrightarrow{O_{2} A}\right) \sin \left(\theta_{2}\right) \vec{i}-\alpha_{2}\left(\overrightarrow{O_{2} A}\right) \cos \left(\theta_{2}\right) \vec{j} \\
& +\omega_{3}^{2}(\overline{A B}) \cos \left(\theta_{3}\right) \vec{i}+\omega_{3}^{2}(\overrightarrow{A B}) \sin \left(\theta_{3}\right) \vec{j} \\
& +\alpha_{3}(\overline{A B}) \sin \left(\theta_{3}\right) \vec{i}-\alpha_{3}(\overline{A B}) \cos \left(\theta_{3}\right) \vec{j}=0
\end{aligned}
$$

The vector equation above constitutes 2 algebraic equations that can be solved for the angular accelerations of the coupler's and follower's angular accelerations: $\alpha_{3}=-1.157 \mathrm{rad} / \mathrm{sec}^{2}$ and $\alpha_{4}=-45.31 \mathrm{rad} / \mathrm{sec}^{2}$.

The acceleration of point $B$ can be calculated: $\overrightarrow{a_{B}}=4.035 \times 10^{3 \vec{i}}+6.956 \times 10^{2} \vec{j}\left(a_{B}=4.095 \mathrm{~m} / \mathrm{sec}^{2}, \angle \overrightarrow{a_{B}}=9.781^{\circ}\right)$.

Next, acceleration analysis of the four bar will be performed through the proposed graphical method as seen in Fig. 10:

(1) $\overline{O^{\prime} A}=\frac{a_{A}}{2 \omega_{3}^{2}}=\frac{\sqrt{\left(\omega_{2}^{2}\left(\overline{O_{2} A}\right)\right)^{2}+\left(\alpha_{2}\left(\overline{O_{2} A}\right)\right)^{2}}}{2 \omega_{3}^{2}}=116.159 \mathrm{~mm}$. $\overrightarrow{a_{A}}$ makes an angle of $14.036^{\circ}$ (i.e. $\tan ^{-1}\left(\frac{\alpha_{2}}{\omega_{2}^{2}}\right)$ ) with $\overrightarrow{\mathrm{O}_{2} \mathrm{~A}} \Longrightarrow \mathrm{O}^{\prime}$ lies at a distance of $116.159 \mathrm{~mm}$ from $A$ along the direction of $\overrightarrow{a_{A}}$.

(2) Copy coupler $\overline{A B}$ from point $A$ and paste at $O^{\prime}$. Call it $\overline{O^{\prime} B^{\prime}}$ and sketch its perpendicular bisector.

(3) Intersect the perpendicular bisector from step 2 with the normal dropped from point $B_{n}$ at distance $\frac{\omega_{4}^{2}\left(\overline{O_{4} B}\right)}{2 \omega_{3}^{2}}=13.995 \mathrm{~mm}$ from $B$. The intersection of the two normals is point $O^{\prime \prime}$.

(4) Angle between $\overline{O^{\prime} B^{\prime}}$ and $\overline{O^{\prime} O^{\prime \prime}}$ gives $\psi=4.967^{\circ}$.

Now all acceleration analysis data are available. For example, we can get the angular acceleration of the coupler link $(\overline{A B})$ as: $\alpha_{3}=\tan (\psi) \omega_{3}^{2}=1.157 \mathrm{rad} / \mathrm{sec}^{2}$. The magnitude of the acceleration of the coupler can be simply measured from Fig. 9 as $a_{B}=2 \omega_{3}^{2} \overline{B O^{\prime \prime}}=(2)(3.64)^{2}(153.82)=4.095 \mathrm{~m} / \mathrm{sec}^{2}$

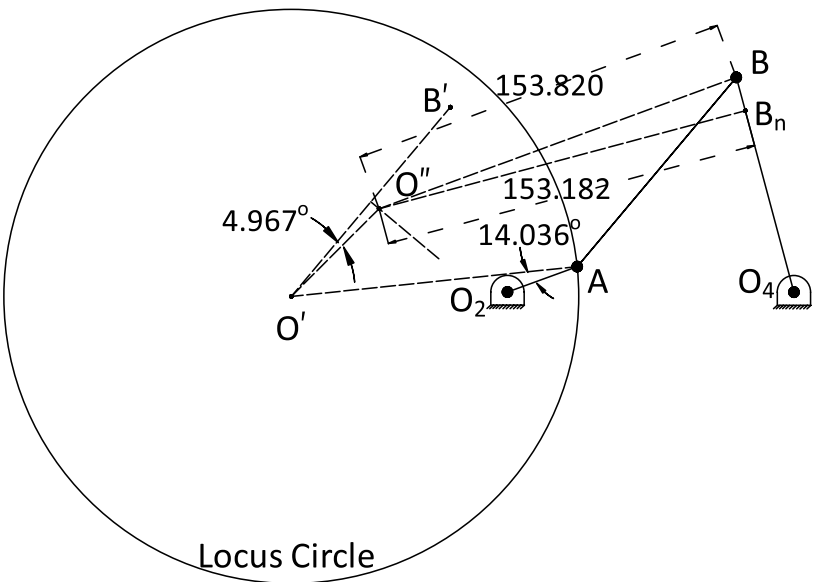

Fig. 10 Graphical constructions to determine $\psi$ for the 4R mechanism

and the angular acceleration of the follower link $\left(\overline{O_{4} B}\right)$ as: $\alpha_{4}=\frac{a_{B}^{t}}{\bar{O}_{4} B}=\frac{2 \omega_{3}^{2}\left(\overline{B_{n} O \prime \prime}\right)}{\overline{O_{4} B}}=\frac{2(3.64)^{2}(153.182)}{90}=45.31 \mathrm{rad} / \mathrm{sec}^{2}$.

The obtained results from the analytical and proposed graphical methods perfectly agree. The instantaneous center of zero acceleration of the coupler link (i.e point $Z$ ) is readily available by rotating the acceleration vector of the driver/coupler joint (i.e. point $A$ ) given along $\overrightarrow{A O^{\prime}}$ or the acceleration vector of the coupler/follower joint (i.e. point $B$ ) given along $\overrightarrow{B O^{\prime \prime}}$ by an angle $\psi=4.967^{\circ}$. Alternatively, point $Z$ can be located as the intersection of the circle of center $O^{\prime}$ and radius $\overline{O^{\prime} A}$ with that of center $O^{\prime \prime}$ and radius $\overline{O^{\prime \prime} B}$. All aforementioned constructions are shown in Fig. 11.

Moreover, the inflection circle can now be easily constructed (see Fig. 12) as it passes through the points $P, Z$ and $Q$ where the latter is obtained as the intersection of the acceleration vector at point $P$ (at an angle $\psi$ from $P Z$ ) with the velocity vector at point $Z$.

\section{Varying the driver's angular acceleration}

The graphical method presented above can be easily extended into a simple and straightforward technique for performing acceleration analysis of four bars (RRRR or RRRP) at different driving angular accelerations. The key to this proposed technique is in locating the intersection of circle $C$ traced by the coupler's $I C_{a}$ and the instantaneous coupler's inflection circle as shown in Fig. 12.

Obviously, from Eq. 6, the location of $Z_{i}$ is dependent on the value of $\alpha_{2}$ and consequently on the value of $\psi_{i}$. In other words, for each value of the driver's angular acceleration $\left(\alpha_{2}\right)$, a unique circle $\left(C_{i}\right)$ as shown in Fig. 2 can be 


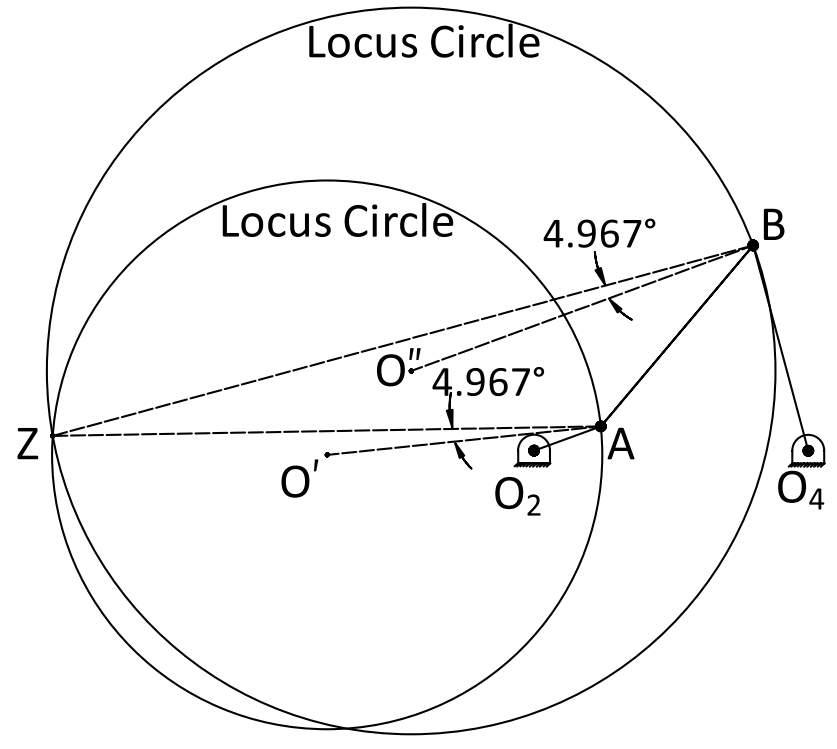

Fig. 11 Graphical constructions to determine the location of the $I C_{a}$ for the $4 \mathrm{R}$ mechanism

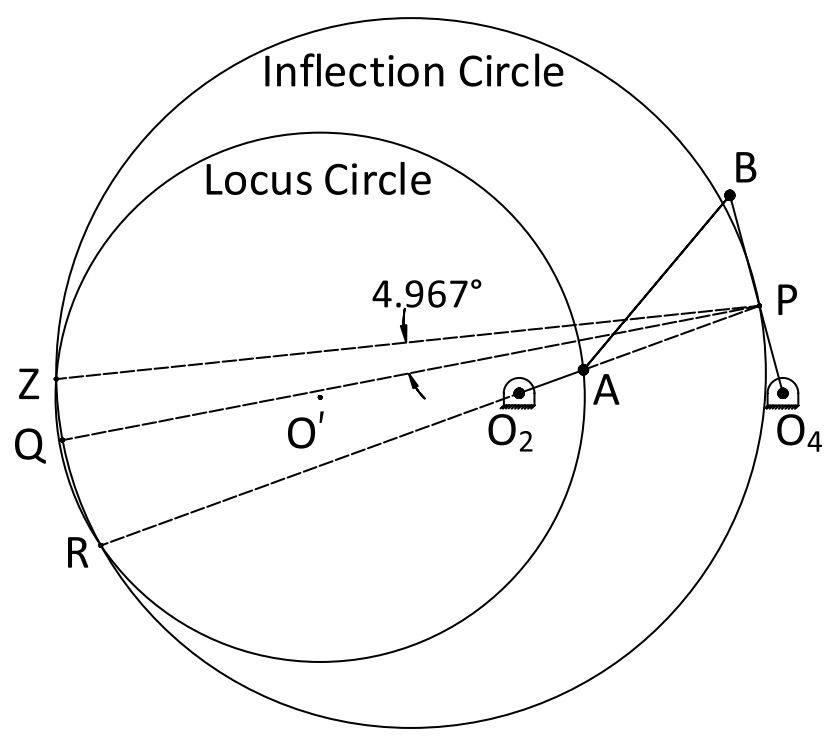

Fig. 12 Inflection circle of the 4R mechanism

constructed and the intersection of that circle with the inflection circle locates point $Z_{i}$ and consequently gives the values of $\psi_{i}$ and $\alpha_{3}$ as explained earlier. In general, these circles intersect at two points, one of them is point $Z_{i}$ which depends on the value of $\alpha_{2}$ (and consequently $\psi_{i}$ ) while the other is a special fixed point $(R)$ located along the direction of the driver's link (Fig. 12). In what follows, an analytical prove is given to locate the second point of intersection $(R)$ between circle $C_{i}$ and the inflection circle.

For point $R$ to be the instantaneous center of zero acceleration of the coupler link (i.e. to coincide with point $Z$ ), the angle of the acceleration vector of point $A$ with respect to the driver $\left(\overline{O_{2} A}\right)$ should be of the same magnitude but opposite direction to $\psi_{i}$. This special circle, unlike all other circles $\left(C_{i}\right)$, will intersect the inflection circle at only one point since $R$ and $Z$ coincide and hence circle $C_{i}$ will be internally tangent to the inflection circle. The value of the driver's angular acceleration $\left(\alpha_{2}\right)$ corresponding to this special circle $C_{i}$ can be found as follows. The value of $\psi_{i}$ at which $R$ is coincident with $Z$ is given by

$\tan \left(\psi_{i}\right)=\frac{\alpha_{2}}{\omega_{2}^{2}}=\frac{\alpha_{3}}{\omega_{3}^{2}} \Longrightarrow \alpha_{2}=\frac{\alpha_{3} \omega_{2}^{2}}{\omega_{3}^{2}}$

But from Eq. 9,

$\overline{Z_{i} A}=\left(\frac{a_{A}}{\omega_{3}^{2}}\right) \cos \left(\psi_{i}\right)=\frac{\sqrt{\left(\overline{O_{2} A} \alpha_{2}\right)^{2}+\left(\omega_{2}^{2} \overline{O_{2} A}\right)^{2}}}{\omega_{3}^{2}} \cos \left(\psi_{i}\right)$

Therefore, Eqs. 17 and 18 give,

$$
\begin{aligned}
\overline{Z_{i} A} & =\overline{O_{2} A}\left(\frac{\omega_{2}}{\omega_{3}}\right)^{2}\left(\sqrt{\left(\frac{\alpha_{3}}{\omega_{3}^{2}}\right)^{2}+1}\right) \cos \left(\psi_{i}\right) \\
& =\overline{O_{2} A}\left(\frac{\omega_{2}}{\omega_{3}}\right)^{2}\left(\sqrt{\tan ^{2}(\psi)+1}\right) \cos \left(\psi_{i}\right) \\
& =\overline{O_{2} A}\left(\frac{\omega_{2}}{\omega_{3}}\right)^{2}
\end{aligned}
$$

The above equation proves that all circles $C_{i}$ pass through point $R$ which is independent of $\psi_{i}$. It is very important to clarify that the aforementioned point $R$ is the instantaneous center of zero acceleration when and only when $\alpha_{2}$ attains the value indicated in Eq. 17. For all other values of $\alpha_{2}$, point $R$ will represent the other point of intersection of circle $C_{i}$ with the inflection circle besides the instantaneous center of zero acceleration $\left(Z_{i}\right)$.

\section{Case study: RRRR mechanism}

The four-bar mechanism in Fig. 9 is revisited herein for acceleration analysis under varying driver's angular acceleration where $\alpha_{2}$ is varied and takes the values $0,10,25$ and $35 \mathrm{rad} / \mathrm{sec}^{2}$ respectively.

Table 1 lists the angular accelerations of the coupler and follower links for different values of the driver's angular acceleration as obtained by the loop closure analytical equations in 16.

The same analysis is now conducted with the graphical method proposed in this work. The first step is to locate point $Z$ (or $R$ ) along $\overline{O_{2} A}$ as per Eq. 19 (Fig. 13). Since $\omega_{2}$ is fixed, as $\alpha_{2}$ is varied, point $O^{\prime}$ will trace line $n$ normal to $\overline{R A}$ 
Table 1 Coupler's and Follower's angular accelerations for varying Driver's angular acceleration obtained by analytical and proposed graphical methods

\begin{tabular}{lllllll}
\hline$\alpha_{2}\left(\mathrm{rad} / \mathrm{sec}^{2}\right)$ & \multicolumn{2}{l}{ Analytical solution } & & \multicolumn{3}{l}{ Proposed graphical solution } \\
\cline { 2 - 3 } & $\alpha_{3}\left(\mathrm{rad} / \mathrm{sec}^{2}\right)$ & $\alpha_{4}\left(\mathrm{rad} / \mathrm{sec}^{2}\right)$ & & $\psi(\mathrm{deg})$. & $\alpha_{3}\left(\mathrm{rad} / \mathrm{sec}^{2}\right)$ & $\alpha_{4}\left(\mathrm{rad} / \mathrm{sec}^{2}\right)$ \\
\hline 0 & 7.964 & -50.397 & & 30.892 & 7.964 & -50.396 \\
10 & 4.316 & -48.362 & & 17.963 & 4.316 & -48.362 \\
25 & -1.157 & -45.31 & & -4.969 & -1.157 & -45.308 \\
35 & -4.805 & -43.276 & & -19.851 & -4.805 & -43.276 \\
\hline
\end{tabular}

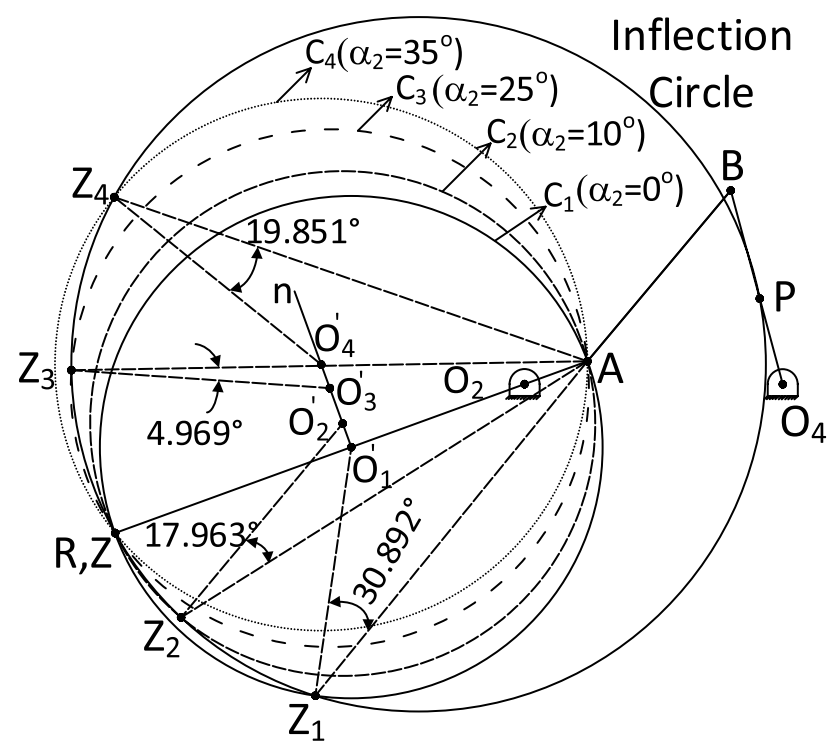

Fig. 13 Locating the coupler's $I C_{a}$ and evaluating its angular acceleration $\alpha_{3}$ for varying Driver's angular acceleration $\alpha_{2}$

at point $O_{1}^{\prime}$. Therefore, for any given value of $\alpha_{2}$, the following simple steps are followed to find the corresponding values of $\alpha_{3}$ and $\alpha_{4}$ :

(1) The circle $C_{i}$ of center $O_{i}^{\prime}$ located at a distance $\frac{\alpha_{2} \overline{\mathrm{O}_{2} \mathrm{~A}}}{2 \omega_{3}^{2}}$ from $O_{1}^{\prime}$ (along $n$ ) and radius $O_{i}^{\prime} A$ is constructed where it intersects the inflection circle at points $R$ and $Z_{i}$ (i.e. $I C_{a}$ of the coupler link)

(2) Having located point $Z_{i}$, the angle $\psi_{i}$ defined by $\angle O_{i}^{\prime} Z_{i} A$ is measured and the value of $\alpha_{3}$ can be easily calculated through Eq. 17

(3) Locate point $O_{i}^{\prime \prime}$ where $\angle O_{i}^{\prime \prime} Z_{i} A$ equals $\psi_{i}$ and calculate $\alpha_{4}=\frac{2 \omega_{3}^{2}\left(\overline{B_{n} O^{\prime \prime}}\right)}{\overline{O_{4} B}}$

The above steps 1 through 3 will be repeated to get $\alpha_{3}$ and $\alpha_{4}$ for any given value of $\alpha_{2}$ as shown in Fig's 13 and 14 . The values obtained from the proposed graphical method are in complete agreement with the values obtained analytically as listed in Table 1.

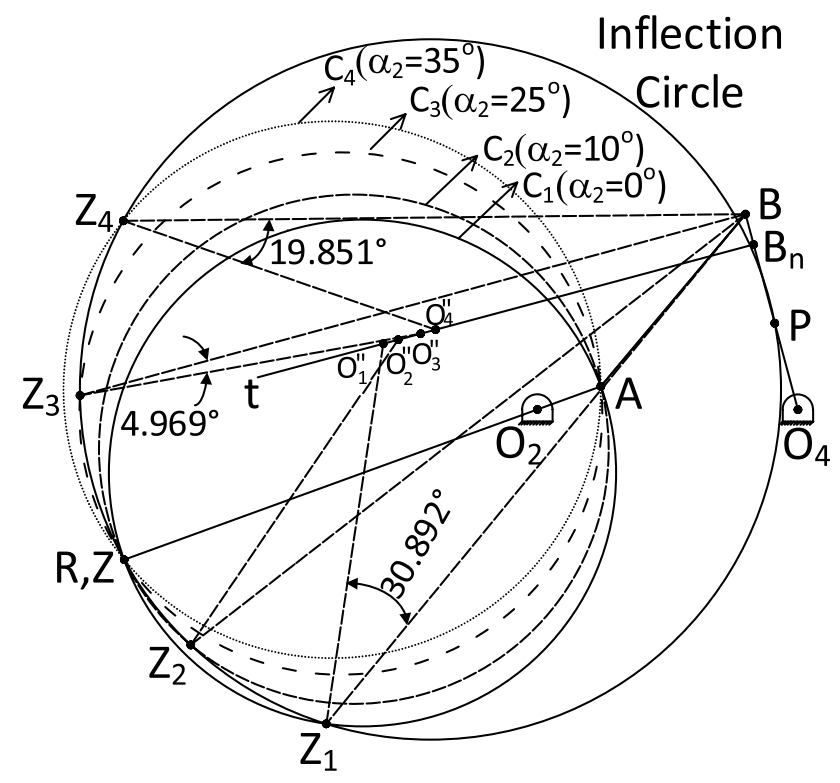

Fig. 14 Locating $O^{\prime \prime}$ and evaluating the Follower's angular acceleration $\alpha_{4}$ for Varying Driver's angular acceleration $\alpha_{2}$

\section{Discussion of results}

As demonstrated in the three case studies of Sects. 4, 5 and 6 , the graphical technique proposed in this work presents a genuine approach for acceleration analysis of four bar mechanisms. One advantage for this new technique is its ability to simultaneously locate the $I C_{a}$ of the coupler link and solve, without further formulations, for the acceleration at any point on the mechanism. This is a prominent advantage over other techniques previously discussed in literature $[10,17,35,41$, $44]$ that require lengthy and additional steps to analyze the kinematics of planar mechanisms after locating the $I C_{a}$. As another advantage, the technique proposed in this study generates the inflection circle of the coupler link in a straightforward manner without the need to formulate Euler-Savary equations as presented in literature $[1,11,19,34]$. The inflection circle is exploited in a genuine approach to easily perform acceleration analysis of four bar linkages with varying driver's acceleration as shown in Sect. 6. Acceleration analysis for the slidercrank and four-bar mechanisms are benchmarked with 
those generated from classical kinematic equations [27] and the results matched with high accuracy (details in Sects. 4, 5 and 6).

\section{Conclusion}

This paper presents a new graphical based method for performing acceleration analysis of planar linkages. The proposed approach requires very few graphical constructions that are sufficient to determine the unknown angular accelerations of all links and the acceleration at any point on the mechanism. In the proposed method and unlike previous work in literature, performing acceleration analysis, locating the instantaneous center of zero acceleration and constructing the inflection circle of the coupler link are all accomplished simultaneously. The ease of construction and potential of the proposed method in obtaining various kinematic properties of planar linkages is believed to be of high added value to the literature of kinematics. As the current study is limited to four bar planar mechanisms with one degree-of-freedom, future development of this work is to extend its applicability to more complex planar and spatial mechanisms with singleor multi-degree-of-freedom.

\section{Compliance with ethical standards}

Conflicts of interest The authors declare that they have no conflict of interest.

Open Access This article is licensed under a Creative Commons Attribution 4.0 International License, which permits use, sharing, adaptation, distribution and reproduction in any medium or format, as long as you give appropriate credit to the original author(s) and the source, provide a link to the Creative Commons licence, and indicate if changes were made. The images or other third party material in this article are included in the article's Creative Commons licence, unless indicated otherwise in a credit line to the material. If material is not included in the article's Creative Commons licence and your intended use is not permitted by statutory regulation or exceeds the permitted use, you will need to obtain permission directly from the copyright holder. To view a copy of this licence, visit http://creativecommons .org/licenses/by/4.0/.

\section{References}

1. Beyer R (1963) The Kinematic Synthesis of Mechanisms. Chapman and Hall, London, England

2. Brunnthaler K, Pfurner M, Husty M (2006) Synthesis of planar four-bar mechanisms. Transac Canadian Soc Mech Eng 30(2):297-313

3. Buckley R, Whitfield E (1949) The eulersavary formula. Mathem Gazette 33(306):297-299
4. Chang Y, Her I (2008) A virtual cam method for locating instant centers of kinematically indeterminate linkages. J Mech Des https://doi.org/10.1115/1.2900720

5. Crowder R (1991) An anthropomorphic robotic end effector. Rob Auton Syst 7(4):253-268

6. Darina H, Peter F, Gabriel B (2016) Kinematical analysis of crank slider mechanism with graphical method and by computer simulation. Am J Mech Eng 4(7), 329-343 . 10.12691/ajme-4-7-18

7. Diab N (2018) A new approach for locating the instantaneous centers of zero velocity for 1-dof planar linkages. In: Proceedings of the ASME International Mechanical Engineering Congress and Exposition (IMECE), IMECE2018-86341. Pittsburgh, Pennsylvania . https://doi.org/10.1115/IMECE2018-86341

8. Diab N, Smaili A (2008) Optimum exact/approximate point synthesis of planar mechanisms. Mech Mach Theory 43(12):1610-1624

9. Diab N, Smaili A (2015) An elitist ants-search based method for optimum synthesis of rigid-linkage mechanisms. In: Proceedings of the ASME International Mechanical Engineering Congress and Exposition (IMECE), pp. 353-359. Houston, Texas . 10.1115/IMECE2015-51374

10. Dicker J, Pennock G, Shigley J (2003) Theory of machines and mechanisms. Oxford University Press, Oxford

11. Dijksman E (1976) Motion geometry of mechanisms. Cambridge University Press, Cambridge, England

12. Dijksman E (1977) Why joint-joining is applied on complex linkages. In: 2nd international symposium on linkages and computer aided design methods, vol. 1, pp. 185-212. Bucuresti

13. Dijksman E (1984) Geometric determination of coordinated centers of curvature in network mechanisms through linkage reduction. Mech Mach Theory 19(3):289-295

14. Erdman A, Sandor G, Kota S (2001) Mechanism design, vol 1. Prentice-Hall, New Jersey

15. Foster D, Pennock G (2003) A graphical method to find the secondary instantaneous centers of zero velocity for the double butterfly linkage. J Mech Des 125:268-274. https://doi. org/10.1115/1.1567313

16. Gregorio RD (2008) An algorithm for analytically calculating the positions of the secondary instant centers of indeterminate linkages. J Mech Des. https://doi.org/10.1115/1.2839008

17. Guiggiani M (2014) The science of vehicle dynamics. Springer NewYork

18. Hain K (1967) Applied kinematics. McGraw-Hill, New York

19. Hall A (1986) Kinematics and linkage design. Waveland Press, Prospect Heights, Illinois

20. Hassan A, Abomoharam M (2014) Design of a single dof gripper based on four-bar and slider-crank mechanism for educational purposes. Procedia CIRP 21:379-384

21. Hassan G (2015) Synthesis of planar mechanisms, part iii: four-bar mechanisms for three coupler-positions generation. Global J Adv Res 2(4):726-734

22. Hassan G, Al-Gamil M, Lashin M (2012) New approach for the synthesis of planar 4-bar mechanisms for 2 coupler-positions generation. New York Sci J 5(10):86-90

23. Kamat G, Hoshing G, Pawar A, Lokhande A, Patunkar P, Hatawalane $S$ (2014) Synthesis and analysis of adjustable planar four-bar mechanism. Int J Adv Mech Eng 6(3):263-268

24. Kung C, Wang L (2009) Analytical method for locating the secondary instant centers of indeterminate planar linkages. Proc Inst Mech Eng Part C: J Mech Eng Sci 223(2):491-502

25. Laribi M, Mlika A, Romdhane L, Zeghloul S (2008) A combined genetic algorithm-fuzzy logic method (ga-fl) in mechanisms synthesis. Mech Mach Theory 39:717-735

26. McDougall R, Nokleby S (2008) Synthesis of grashof fourbar mechanisms using particle swarm optimization. In: 
Proceedings of IDETC/CIE 2008 ASME 2008 International Design Engineering Technical Conferences\&Computers and Information in Engineering Conference. Brooklyn, New York, USA . 10.1115/DETC2008-49631

27. Norton R (2008) Kinematics and dynamics of machinery. McGraw-Hill, NewYork

28. Ouyang P, Zhang W (2005) Force balancing of robotic mechanisms based on adjustment of kinematic parameters. J Mech Des 27:433-440. https://doi.org/10.1115/1.1864116

29. Pennock G (2003) Path curvature of a geared seven-bar mechanism. In: Proceedings of the 11 th World Congress in Mechanism and Machine Science. Tianjin, China

30. Pennock G, Kinzel E (2004) Graphical technique to locate the center of curvature of a coupler point trajectory. J Mech Des 126:1000-1005. https://doi.org/10.1115/1.1798091

31. Romaniak K (2009) Sensitivity analysis of planar mechanisms. J Theoretical Appl Mech 47(2):473-482

32. Rosenauer N, Willis A (1953) Kinematics of mevchanisms. Associated General Publications, Sydney, Australia

33. Saha D, Sanfui S, Kabiraj R, Das S (2014) Design and implementation of a 4-bar linkage gripper. IOSR J Mech Civil Eng 11(5):61-66

34. Sandor G, Xu Y, Weng T (1990) A graphical method for solving the euler-savary equation. Mech Mach Theory 25(2):141-147

35. Schiller R (1965) Method for determining the instantaneous center of acceleration for a given link. J Appl Mech 32(1):217-218

36. Shahhosseini $M$, Rastegari $R$, Abbasi $R$ (2016) A new 6 dof robotic arm with linkage motion mechanism and actuators placed in base. Int J Robot Autom 5(1):35-48

37. Singh P, Kumar A, Vashisth $M$ (2013) Design of a robotic arm with gripper end effector for spot welding. Univ J Mech Eng 1(3):92-97

38. Smaili A, Diab N (2007) A new approach to shape optimization for closed path synthesis of planar mechanisms. Mech and Mach Theory 129(9):941-948

39. Smaili A, Diab N (2007) Optimum synthesis of hybrid-task mechanisms using ant-gradient search method. Mech Mach Theory 42(1):115-130

40. Sukumaran S, Deivanathan R (2014) Optimum linkage for biped mechanism. Procedia Engineering pp. 1322-1331 . 10.1016/j. proeng.2014.12.412
41. Sun H, Liu T (2009) Analysis of instantaneous center of zero acceleration of rigid body in planar motion. Mod Appl Sci 3(4):191-195

42. Tari H, Su H (2010) Complete solution to the eight-point path generation of slider-crank four-bar linkages. J Mech Des. https ://doi.org/10.1115/1.4001878

43. Telegenov K, Tlegenov Y, Hussain S, Shintemirov A (2015) Preliminary design of a three-finger underactuated adaptive end effector with a breakaway clutch mechanism. J Robot Mech 27(5):496-503

44. Tesar D, Curran C (2011) The analytical formulations for vehicle motion planning. Mod Mach Sci J 04:284-293

45. Tischler C, Hunt K, Samuel A (1998) On optimizing the kinematic geometry of a dexterous robot finger. Int J Robot Res 17(10):1055-1067

46. Torodov T (2015) Synthesis of four bar mechanisms as function generators by freudenstein - chebyshev. J Robot Mech Eng Res 1(1):1-6

47. Uchida T, McPhee J (2012) Using grbner bases to generate efficient kinematic solutions for the dynamic simulation of multiloop mechanisms. Mech Mach Theory 52:144-157. https://doi. org/10.1016/j.mechmachtheory.2012.01.015

48. Waldron K, Kinzel G (1999) Kinematics, dynamics, and design of mmachinery. Wiley, New York

49. Wolbrecht, E., Reinkensmeyer, D., Perez-Gracia, A.: Single degreeof-freedom exoskeleton mechanism design for finger rehabilitation. In: IEEE International Conference on Rehabilitation Robotics, pp. 1-6. Zurich (2011). 10.1109/ICORR.2011.5975427

50. Wu Y, Gosselin C (2002) Kinematic analysis of spatial 3-dof parallelepiped mechanisms. Advances in Robot Kinematics pp. 423-432

51. Yan H, Hsu M (1992) An analytical method for locating velocity instantaneous centers. In: Proceedings of the $22 \mathrm{nd}$ Biennial ASME Mechanisms Conference, vol. 47, pp. 353-359. Scottsdale, Arizona . 10.1109/ICORR.2011.5975427

52. Zarkandi S (2010) Geometrical methods to locate secondary instantaneous poles of single-dof indeterminate spherical mechanisms. J Mech Eng 41(2):80-88

Publisher's Note Springer Nature remains neutral with regard to jurisdictional claims in published maps and institutional affiliations. 